\title{
Human Cone Pigment Expressed in Transgenic Mice Yields Altered Vision
}

\author{
Gerald H. Jacobs, ${ }^{1}$ John C. Fenwick, ${ }^{1}$ Jack B. Calderone, ${ }^{1}$ and Samir S. Deeb ${ }^{2}$ \\ ${ }^{1}$ Neuroscience Research Institute and Department of Psychology, University of California, Santa Barbara, California \\ 93106, and 2Departments of Medicine and Genetics, University of Washington, Seattle, Washington 98195
}

\begin{abstract}
Genetically driven alterations in the complement of retinal photopigments are fundamental steps in the evolution of vision. We sought to determine how a newly added photopigment might impact vision by studying a transgenic mouse that expresses a human cone photopigment. Electroretinogram (ERG) measurements indicate that the added pigment works well, significantly changing spectral sensitivity without deleteriously affecting the operation of the native cone pigments. Visual capacities of the transgenic mice were established in behavioral tests. The new
\end{abstract}

pigment was found to provide a significant expansion of the spectral range over which mice can perceive light, thus underlining the immediate utility of acquiring a new photopigment. The transgenic mouse also has the receptor basis for a novel color vision capacity, but tests show that potential was not realized. This failure likely reflects limitations in the organizational arrangement of the mouse retina.

Key words: cone photopigments; transgenic mice; visual sensitivity; color vision; gene coexpression; retina
Photopigments have effective fixed spectral bandwidths across which the efficiency of absorption varies significantly. Examination of the great variety of photopigments found in contemporary species suggests that two evolutionary strategies have been used to accommodate this restriction in absorption bandwidth to an array of different visual demands. One is to spectrally position the photopigment to optimize some visual capacities. A classic example is the correlation between the spectral positioning of rod pigments found in deep sea fishes and the spectral distribution of light in their marine environments (Douglas et al., 1998). A second strategy is to add new photopigment types. Two general advantages may accrue from adding new photopigments: (1) the available spectral window can be significantly expanded, and (2) if there are appropriate nervous system connections the signals originating from different types of photopigment may yield color vision or other wavelength-specific behaviors. The addition of a third type of cone pigment and the emergence of trichromatic color vision in primates from a background of mammalian dichromacy provides a well known example of the utility of this second strategy (Jacobs, 1993).

Although the genetic mechanisms underlying shifts in photopigment spectra and the addition of new pigments have been much studied in recent years (Nathans, 1987; Deeb and Motulsky, 1996; Neitz and Neitz, 1998), there is little understanding of how the presence of novel photopigments can yield new visual capacities. In one group of animals that have been studied, the New World monkeys, it appears that newly added photopigments were probably immediately translated into expanded visual sensitivity and additional color vision capacities (Jacobs, 1998). Other cases could presumably also require some modifications of neural architecture for maximum exploitation of the new potential for

\footnotetext{
Received Dec. 9, 1998; revised Feb. 5, 1999; accepted Feb. 8, 1999.

This work was supported by Grants EY02052 (G.H.J.) and EY08395 (S.S.D.) from the National Eye Institute. We thank Kris Krogh and Alexander Nugent for technical assistance.

Correspondence should be addressed to Dr. Gerald H. Jacobs, Neuroscience Research Institute, University of California, Santa Barbara, CA 93106.

Copyright (C) 1999 Society for Neuroscience $\quad 0270-6474 / 99 / 193258-08 \$ 05.00 / 0$
}

capturing light. As a step toward allowing an experimental examination of these issues, in a recent investigation a gene encoding a human cone photopigment was introduced into the mouse genome (Shaaban et al., 1998). The human transgene was abundantly expressed in mouse cones and the pigment was found to be efficient at transducing light. We have now examined the manner in which the activity of this new photopigment is reflected in signals in the outer retina and have conducted behavioral experiments to determine to what extent the mouse visual system is able to capitalize on the presence of a newly acquired photopigment.

\section{MATERIALS AND METHODS}

Subjects. Male mice, both transgenic animals and wild-type controls (C57/BL6), were studied. Details of the methods used to produce the transgenic mice and to establish expression of the foreign photopigment have been published previously (Shaaban et al., 1998). In brief, mice transgenic for a human long-wavelength $(\mathrm{L})$ photopigment gene were generated by microinjection of fertilized mouse eggs. The pattern of inheritance of the transgene was consistent with autosomal integration of the gene. The experiments reported here were all conducted on descendants of a mouse having two copies of the transgene. Tissue-specific expression of the transgene was confirmed by performing reverse transcription and PCR amplification on RNA obtained from whole eyes, brain, and liver. Immunocytochemical analysis showed that the human L-pigment was coexpressed with native pigment(s) in the outer segments of $\sim 80 \%$ of all mouse cones.

Electroretinogram measurements. There were two sets of experiments. Electroretinogram (ERG) measurements were made to examine outer retinal signals initiated by cone activity, and behavioral tests of visual discrimination were conducted to provide a specific examination of the contributions of the human L-cone pigment to mouse vision.

ERG spectral measurements were made using a flicker photometric procedure that is fully described elsewhere (Jacobs et al., 1996). Briefly, light pulses derived from a three-beam optical system were presented to the eye in Maxwellian view (59 $9^{\circ}$ circular spot). The optical system was mounted on an adjustable platform that could be positioned so that the beam entered the eye along the optic axis. At its focal point, the beam from the optical system was smaller than the size of the fully dilated pupil of the mouse; the latter is estimated to have a diameter of $2 \mathrm{~mm}$ (Remtalla and Hallett, 1985). The photometer test light was from a monochromator (10 nm half-energy passband). Its intensity was controlled through rotation of a circular, 3.0 log unit neutral-density wedge 
positioned in the light pathway. The photometer reference light and an additional adaptation light constituted the other two beams. These originated from tungsten-halide lamps, and their content was varied through the use of neutral-density step filters and interference filters (Optical Thin Films, $10 \mathrm{~nm}$ halfband). All three light sources were underrun at 11 $\mathrm{V}$ from regulated DC power supplies. High-speed mechanical shutters were used to control the timing of test and reference lights. Light intensities were measured in the plane of pupil using a suppliercalibrated photodiode (Pin-10 DF, United Detector Technology).

To record ERGs, mice were anesthetized with an intramuscular injection of a mixture of xylazine hydrochloride $(6.7 \mathrm{mg} / \mathrm{kg})$ and ketamine hydrochloride $(67 \mathrm{mg} / \mathrm{kg})$. The pupil of the test eye was dilated by topical application of a mixture of $0.2 \%$ phenylephrine hydrochloride and $0.02 \%$ cyclopentolate hydrochloride (Olsson et al., 1992). The mouse was positioned on a heating pad in a head restraint to allow alignment of the eye with the optical system. ERGs were differentially recorded from a pair of stainless-steel ring electrodes. One ring was against the cornea, and the other was positioned farther back on the conjunctiva. Each ring contacted the eye through a layer of artificial tears. A ground electrode was placed in the mouth. All recordings were made in an illuminated room (ambient illuminance at the test eye $=300$ lux). ERGs were generated by an interleaved train of square-wave pulses from the test and reference lights, each modulated with a $25 \%$ duty cycle at $12.5 \mathrm{~Hz}$. The effectiveness of test and reference lights were equated by adjusting the intensity of the test light until the ERG it produced was equivalent to that produced by the constant reference light. The signal processing procedure has been described elsewhere (Jacobs et al., 1996). ERG photometric equations were made by iteratively adjusting the intensity of the test light while recording the averaged responses to the last 50 of a total of 70 stimulus cycles. The wedge density values at the points of equation were recorded to a precision of $0.01 \log$ unit. Equations were made for each stimulus on two separate occasions during an experiment, and these values were subsequently averaged.

Visual discrimination. Visual capacities were determined using a threealternative, forced-choice discrimination. The test apparatus has been described previously (Jacobs, 1983, 1984). The mouse viewed three circular test panels (diameter and center-center distance $=2.5 \mathrm{~cm}$ ) positioned in a line along one wall of a small test chamber. The panels were transilluminated by lights originating from an optical system located outside the chamber. The system has two sources. One is a tungstenhalide lamp that was used to diff usely and equally illuminate each of the panels (background lights). The other source is an Instruments SA (Model H-10) grating monochromator (half-energy passband of $16 \mathrm{~nm}$ ) with a $75 \mathrm{~W}$ xenon lamp. An automated mirror system was used to direct the light from this source (the test light) to any one of the three panels through three diffusing ports. Depending on the experiment being conducted, the test light either was added to the background light or it completely replaced the background light. The interior of the test chamber was diffusely illuminated by a ceiling-mounted fluorescent tube (100 lux).

Through a shaping procedure mice were trained to detect the panel on which the test light appeared. They signaled their choice by touching the panel. Correct choices were reinforced by delivery of a highly palatable fluid (Soymilk, West Soy Plus Plain) that was automatically pumped in increments of $0.028 \mathrm{ml}$ from feeder tubes mounted directly above each panel. Over successive test trials the location of the test light was randomly alternated across the three panels. The nature of the difference between lights on the positive and negative panels was systematically varied to allow examination of several visual capacities as described below. Each test trial was signaled by the occurrence of a cueing tone; the tone terminated when the animal responded or after $15 \mathrm{sec}$ without a response. Intertrial duration was $6 \mathrm{sec}$. A penalty time was used such that the onset of a test trial was delayed by a period of $5 \mathrm{sec}$ after any adventitious between-trial responses. A noncorrection procedure was used. All aspects of stimulus presentation, reinforcement delivery, and response monitoring were computer-controlled. The mice were tested daily and fed standard lab food after each test session in an amount sufficient to hold their weight at a constant level.

The mice were free to move about the test chamber during the experiment. This makes difficult a precise specification of the stimuli. The floor of the test chamber is adjustable in height, and it was set so that the centers of the test panels were at approximately the height of a mouse eye when the animal was in a normal standing posture. Trained subjects were observed with a video camera in an attempt to estimate their average positioning relative to the test panels during the performance of

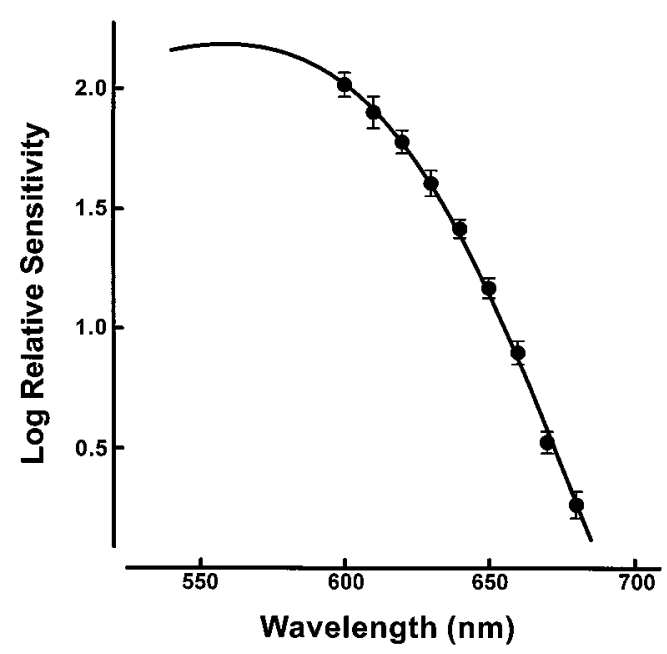

Figure 1. The long-wavelength portion of the spectral sensitivity function of the transgenic mouse. The data points are average sensitivity values for six animals $( \pm 1 \mathrm{SD})$ obtained from ERG flicker photometric measurements. The data obtained from individual animals were not normalized before the calculation of the deviations and that same convention holds for other figures in this report. The curve is that for a photopigment absorption spectrum having peak sensitivity at $556 \mathrm{~nm}$. It was obtained by shifting a photopigment nomogram curve (Dawis, 1981) along a wavenumber axis in successive steps of $1 \mathrm{~nm}$ until the best least-squares fit to the data array was obtained.

actual discriminations. We concluded that, depending on the position of the mouse, the effective stimulus could vary in angular size over a range from $\sim 14$ to $60^{\circ}$, the average probably being closer to the latter value. Our experience with rodent subjects in discrimination situations like this is that they can and sometimes do develop distinctly idiosyncratic viewing behaviors. Nevertheless, the reinforcement contingencies are such that they would be expected to lead subjects to adopt viewing strategies that maximize their performance. The relatively small variation in performance observed across subjects (see Fig. 4) suggests that this in fact happened.

\section{RESULTS}

\section{Cone photopigments in the transgenic mouse}

The L-pigment of the transgenic mouse is one of the two common polymorphic versions of the human L-pigment (Winderickx et al., 1992; Neitz et al., 1993). As studied in artificial expression systems, measurements of this pigment have yielded two different estimates of its $\lambda_{\mathrm{MAX}}: 552$ and $556 \mathrm{~nm}$ (Merbs and Nathans, 1993; Asenjo et al., 1994). To obtain the best estimate of the spectral position of that pigment as expressed in mouse cones, we measured the long-wavelength limb of the spectral sensitivity function using ERG flicker photometry. A $630 \mathrm{~nm}$ reference light $(1.1 \times$ $10^{15}$ quanta $\cdot \sec ^{-1} \cdot \mathrm{sr}^{-1}$ ) was used to minimize contributions from native mouse pigments. At that wavelength, the sensitivity of the mouse middle-wavelength $(\mathrm{M})$ cone pigment has declined to a value that is $<2 \%$ of its peak sensitivity. For a similar reason the test lights were restricted to long wavelengths only (from 580 to $680 \mathrm{~nm}$ in steps of $10 \mathrm{~nm}$ ). Figure 1 shows spectral sensitivity so measured for six transgenic mice. Note the small size of the inter-animal variation. The photopigment absorption function (Fig. 1, continuous line) that best accounts for this array of sensitivity values has a $\lambda_{\mathrm{MAx}}$ of $556 \mathrm{~nm}$. That peak value is hereafter taken as the best estimate of the spectral position of the L-pigment in the transgenic mouse. Figure 2 summarizes the spectra of the three cone photopigments in this transgenic mouse. In addition to the L-pigment, there are two native cone pigments, 


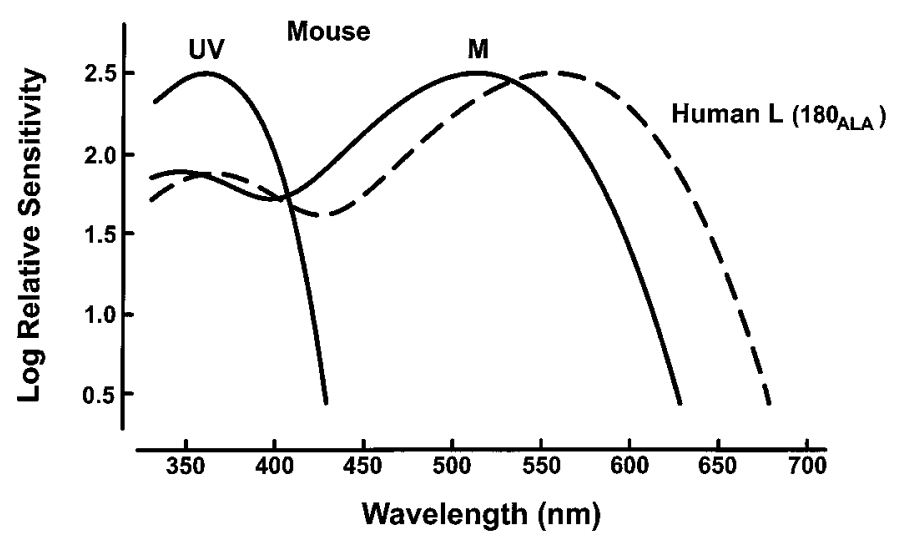

Figure 2. Schematic representation of the spectral sensitivity curves for the three cone pigments of the transgenic mouse. The native pigments ( $U V$ and $M$ ) have respective peak values of 360 and $512 \mathrm{~nm}$. The peak of the transgenic pigment (Human L) is $556 \mathrm{~nm}$. The shapes of the curves were generated from photopigment nomograms (Dawis, 1981). The spectral positioning and shapes of the $\beta$ peaks for M- and L-pigments are those suggested by Palacios et al. (1998).

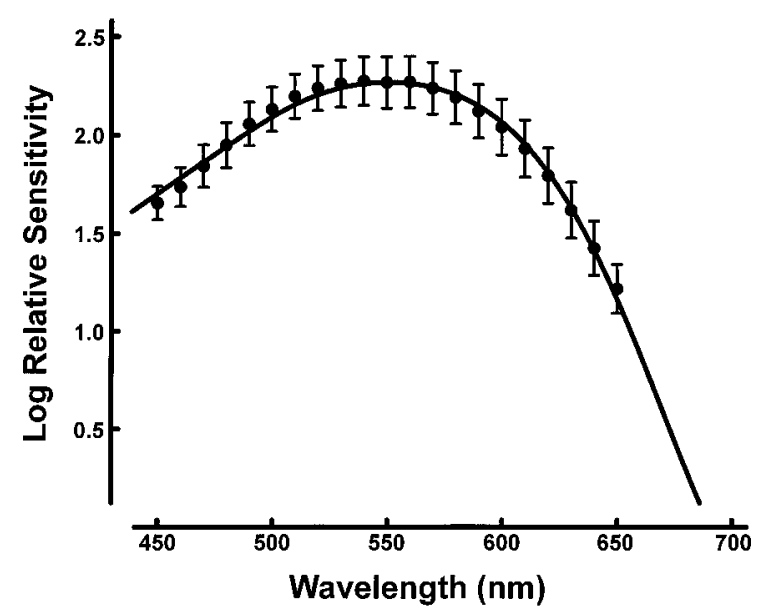

Figure 3. Cone spectral sensitivity functions for transgenic mice. The values (means for 12 animals $\pm 1 \mathrm{SD}$ ) were obtained from ERG flicker photometric measurements. The sensitivity value plotted at $550 \mathrm{~nm}$ corresponds to a light intensity of $2.93 \times 10^{13}$ photons $\cdot \mathrm{sec}^{-1} \cdot \mathrm{sr}^{-1}$. The curve is the best-fitting linear summation of two photopigment absorption curves having respective peak values of $512(15 \%)$ and $556(85 \%)$.

one with the peak absorption $\left(\lambda_{\mathrm{MAX}}\right)$ in the ultraviolet (UV) at $\sim 360 \mathrm{~nm}$ and an M-pigment with a $\lambda_{\mathrm{MAX}}$ of $\sim 509-512 \mathrm{~nm}$ (Jacobs et al., 1991; Lyubarsky et al., 1998).

\section{M/L spectral sensitivity}

ERG measurements of cone spectral sensitivity were made in 11 transgenic mice. Sensitivity was determined over the wavelength range from 450 to $650 \mathrm{~nm}$ at steps of $10 \mathrm{~nm}$. The pulse rate of the photometer was $12.5 \mathrm{~Hz}$, and the reference light was achromatic ( $2450 \mathrm{~K})$. In Figure 3, the solid circles are mean sensitivity values $( \pm 1 \mathrm{SD})$ for the group. The variation between animals was quite small (mean SD value for the 21 test wavelengths $=0.123 \mathrm{log}$ unit). The shape of the sensitivity curve clearly indicates combined contributions from signals originating in the native M-pigment and the transgenic L-pigment. To provide an index of that signal combination, we assumed that contributions from the two pigments to the ERG flicker response are linearly summed. Photopigment absorption spectra having peak values of 512 and

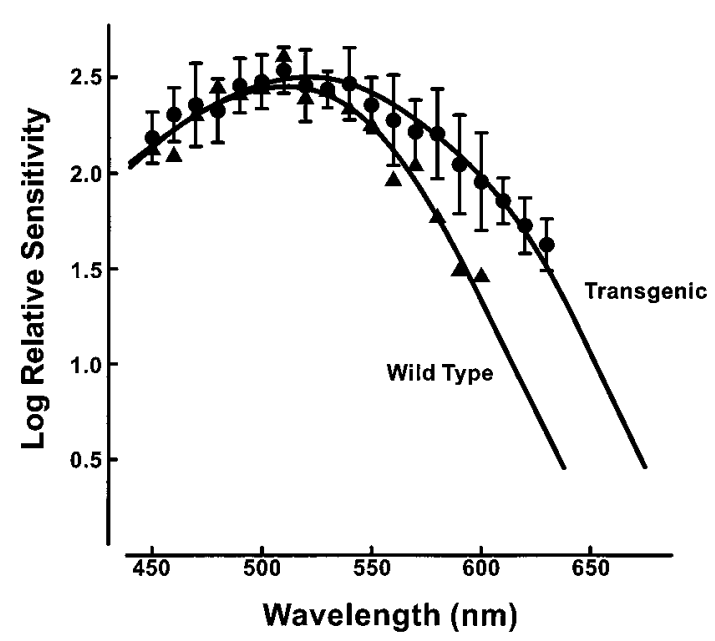

Figure 4. Spectral sensitivity functions for six transgenic mice (mean values $\pm 1 \mathrm{SD}$ ) and one wild-type mouse as determined in an incrementthreshold discrimination task. The sensitivity value for $500 \mathrm{~nm}$ corresponds to a panel light intensity of $1.3 \times 10^{11}$ photons $\cdot \mathrm{sec}^{-1} \cdot \mathrm{mm}^{2-1}$. The curve drawn through the data for the wild-type mouse is a photopigment absorption curve having a $512 \mathrm{~nm}$ peak. The curve fit to the results from the transgenic mice is the best-fit linear summation of two photopigment absorption curves: $512 \mathrm{~nm}(68 \%)+556 \mathrm{~nm}(32 \%)$.

$556 \mathrm{~nm}$ were added in varying proportions (in relative steps of $1 \%$ ) to determine the combination providing the best fit to the data array. The curve in Figure 3 is the best fitting combination: $14 \%(512)+86 \%(556)$.

Photopic spectral sensitivity functions were measured in behavioral experiments for six transgenic animals. An incrementthreshold procedure was used in which the three panels were steadily illuminated with an achromatic light (color temperature $=5350 \mathrm{~K}$ ), and monochromatic light was added to one of these panels during the test trial. Over trials the intensity of the test light was varied in steps of $0.3 \log$ units descending from a level at which the animal showed high levels of discrimination ( $80 \%$ correct or greater) down to an intensity that produced chance performance. The test light was varied in $10 \mathrm{~nm}$ steps from 450 to $630 \mathrm{~nm}$. Performance measures were accumulated over daily test sessions until a total of at least 100 test trials had been run at each wavelength/intensity combination. From these cumulated values psychometric functions were constructed by plotting mean percentage correct as a function of stimulus intensity. These averaged data points were then fit to a logistic function having asymptotes of 100 and $33 \%$ correct with the variance and mean as free parameters. The function providing the best least-squares fit to the data set was determined. From these functions, thresholds were subsequently computed as the stimulus intensity required to yield performance corresponding to the 99\% upper-confidence level.

The behavioral spectral sensitivity functions obtained when the background lights were set to a luminance of $13.2 \mathrm{~cd} / \mathrm{m}^{2}$ are summarized in Figure 4. The solid circles are mean values $( \pm 1$ $\mathrm{SD})$ for six transgenic mice. The individual variation in the increment threshold measurements was quite similar to that seen for the ERG measurements (mean SD across all test wavelengths $=0.14 \log$ unit). The fitted function (continuous line) was determined in the same manner as for the ERG spectral data, i.e., by seeking the best-fitting summative combination of pigment absorption curves having respective peak values of 512 and 556 $\mathrm{nm}$. As can be seen, the combination $512(68 \%)+556(32 \%)$ 
provides a good account of the spectral sensitivity function. Shown for comparison in Figure 4 is the spectral sensitivity function (triangles) for a single wild-type mouse determined in exactly the same manner as for the transgenic mice. The curve fit to the latter data are the absorption spectrum for a single pigment with a $\lambda_{\text {MAX }}$ of $512 \mathrm{~nm}$. Comparison of the two curves makes clear the very substantial increase in sensitivity in the long wavelengths provided by the L-pigment in the transgenic mouse.

In a second experiment, complete spectral sensitivity functions (over a range from 450 to $620 \mathrm{~nm}$ ) were additionally obtained for four of the transgenic mice with the background light levels increased to luminance of $26.9 \mathrm{~cd} / \mathrm{m}^{2}$. Those functions (data not shown) were in no way qualitatively different from those of Figure 4. At this higher background light level, the combination of the two pigment absorption curves needed to best fit the averaged spectral sensitivity function were very slightly different: $(61 \%)$ $512+(39 \%) 556$. Finally, spectral sensitivity functions were also measured over the range of 450 to $630 \mathrm{~nm}$ for two transgenic mice with a dimmer background light $\left(6.7 \mathrm{~cd} / \mathrm{m}^{2}\right)$. Again, the spectral sensitivity functions were similar to those of Figure 4; the best combined fit of the two cone pigments to this function was $(75 \%)$ $512+(25 \%) 556$.

\section{Sensitivity to UV light}

Although our interest in these experiments was focused on the effects of the added L-pigment, we also wanted to determine whether the presence of this new pigment might exert some effect on the operational integrity of the native pigments. The substantial spectral overlap of the L-pigment with native M-pigment makes such a determination difficult (Fig. 2). It is somewhat more straightforward to do this in the case of sensitivity to the UV. Accordingly, we measured spectral sensitivity down into the UV range using both ERG and behavioral discrimination. Figure 5 (top) shows ERG spectral sensitivity functions obtained from a transgenic and a control animal. There is clear evidence for robust contribution to the spectral sensitivity function by the UV cone in both animals. Figure 5 (bottom) also shows behaviorally determined spectral sensitivity functions for two transgenic mice and a single control animal. Although there is relatively much lower sensitivity in the UV wavelengths for behavioral than for ERG measurements, there is no obvious difference between the transgenic and the wild-type animals. We return below to the issue of the disparity in UV sensitivity seen in ERG and behavioral tests.

A comparison was also made between the amplitudes of the ERG cone-based signals in transgenic and wild-type mice. Amplitudes were measured using a stimulus flickering at $12.5 \mathrm{~Hz}$ at three test wavelengths: 390, 500, and $660 \mathrm{~nm}$. These three lights were presented at the maximum intensity available from the monochromator $\left(1.87 \times 10^{13}, 2.79 \times 10^{14}, 4.77 \times 10^{14}\right.$ quanta $\cdot \sec ^{-1} \cdot \mathrm{sr}^{-1}$, respectively), with the responses averaged for five separate presentations, each of which consisted of 50 flashes. The bar graph in Figure 6 summarizes these results for 10 transgenic and 6 control animals. Although there is considerable individual variation, the transgenic and control mice showed no significant differences in responsivity to 390 and $500 \mathrm{~nm}$ lights. Not surprisingly, however, the presence of the L-pigment made the transgenic mice much more responsive to the $660 \mathrm{~nm}$ test light. Both the spectral sensitivity and the amplitudes recorded to UV test lights would be expected to be significantly dependent on activity generated by the mouse UV pigment. There seem to be no clear differences in this regard for transgenic and control mice.

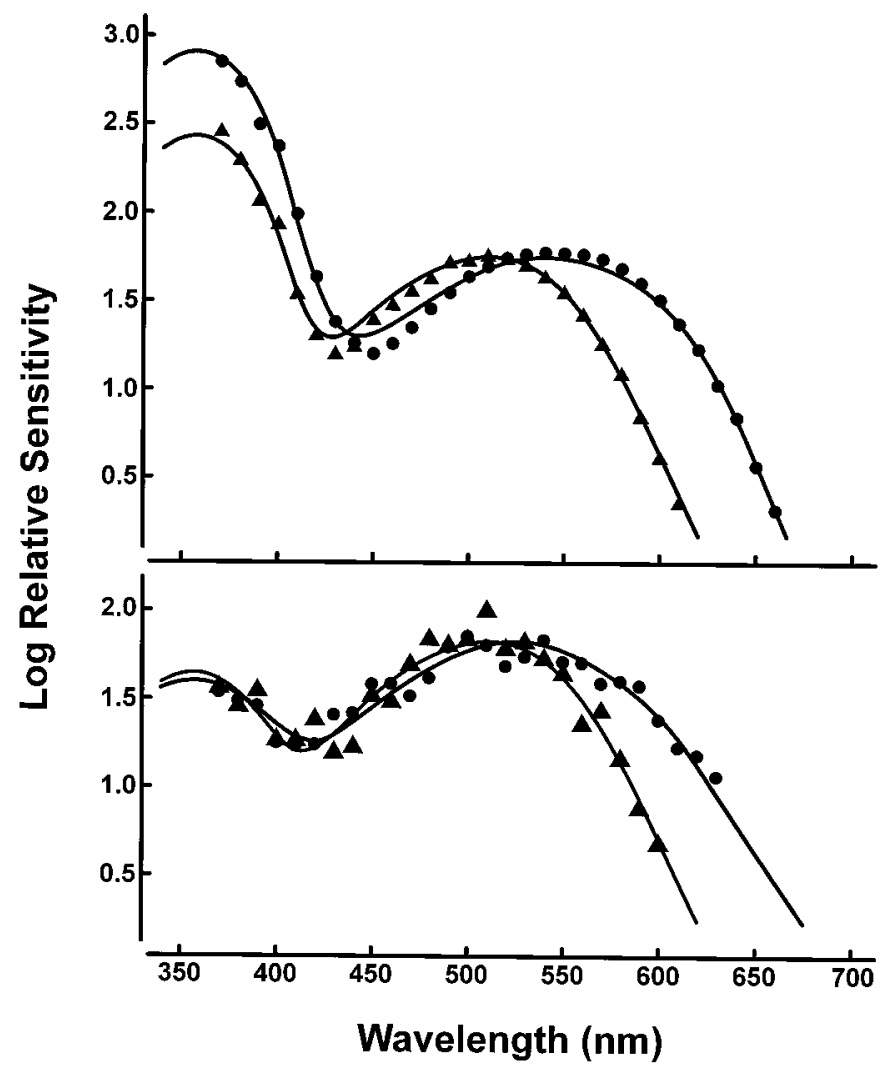

Figure 5. Full spectral sensitivity functions for wild-type $(\boldsymbol{\Delta})$ and transgenic (@) mice as determined with ERG flicker photometry (top, 1 animal each) and behavioral increment-threshold measurements (bottom, 2 transgenic mice, 1 wild-type mouse). The continuous curves are linear summations of the photopigment spectra shown in Figure 2.

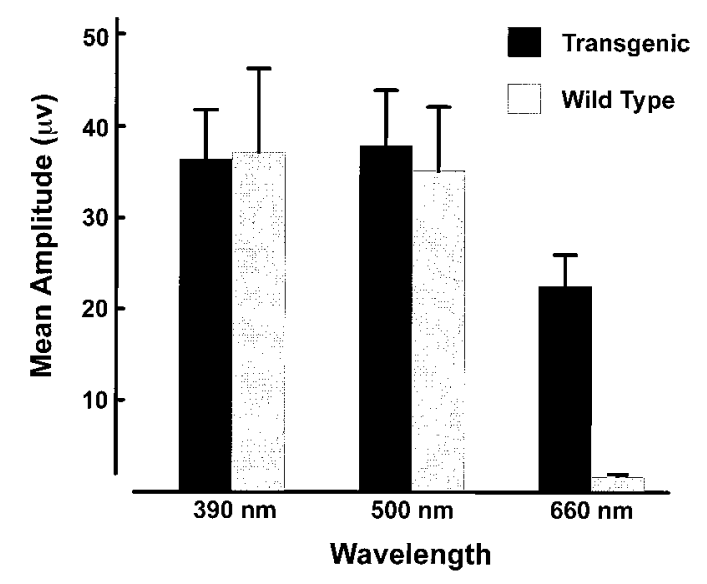

Figure 6. Mean ERG response amplitudes obtained from transgenic $(n=10)$ and wild-type mice $(n=6)$ for three test wavelengths (error bars represent 2 SEM). The stimuli were monochromatic lights flickering at $12.5 \mathrm{~Hz}$.

\section{Spectral limits}

It is clear from the above that the presence of the added L-cone pigment significantly enhances sensitivity to long wavelength lights in the transgenic mice. To document that effect, we determined the spectral limits for these animals. For this, mice were required to discriminate the presence of a monochromatic light added to a dim background light $\left(0.41 \mathrm{~cd} / \mathrm{m}^{2}\right)$. The wavelength of 


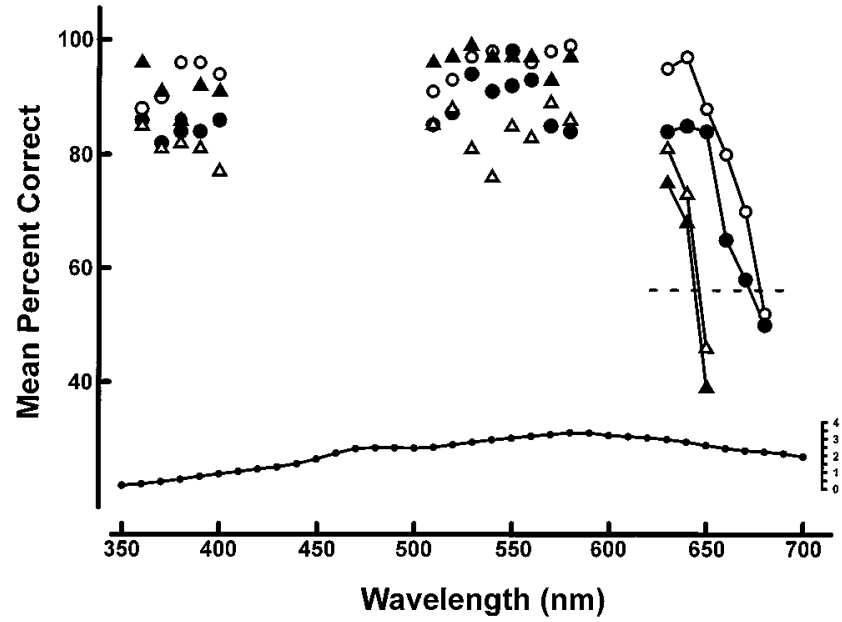

Figure 7. Top, Spectral limits determinations for two transgenic (circles) and two wild-type (triangles) mice. The plotted values represent the average discrimination performance on 100 test trials at each tested wavelength. The dashed line indicates threshold performance. Bottom, The intensities of the test stimuli used as measured with the detector (surface area $=1 \mathrm{~cm}^{2}$ ) placed against the stimulus panels. The ordinate values are quanta $\cdot \mathrm{sec}^{-1} \cdot \mathrm{mm}^{-2} \times 10^{12}$ ).

the monochromatic light was varied across the spectrum to allow a determination of the limits of sensitivity. The monochromatic lights were presented at a single intensity, the maximum available at that wavelength from the source monochromator. The variation in intensity of the test light across the spectrum is shown at the bottom of Figure 7. Two transgenic and two wild-type mice were trained to discriminate the presence of the test light. The test wavelength was varied in $10 \mathrm{~nm}$ steps over the following ranges: $360-410,510-580$, and $630-680 \mathrm{~nm}$. Once the mice were fully trained, their performance on a total of 100 test trials was accumulated at each of the wavelengths. The average performance for each of the four animals is plotted in Figure 7. There was no significant variation among these animals for any wavelengths shorter than $630 \mathrm{~nm}$. However, at wavelengths longer than that, the two types of mice diverged in performance. The transgenic mice (circles) were able to see much longer wavelength lights than the control animals (triangles). For a criterion level of performance ( $57 \%$ correct; Fig. 7 , dashed line), the wild-type mice failed at $645 \mathrm{~nm}$, whereas the transgenic animals were successful out to a wavelength of $676 \mathrm{~nm}$. The presence of the L-pigment has expanded the spectral window by at least $30 \mathrm{~nm}$.

\section{Effects of exposure to chromatic light}

The M- and L-pigments in the transgenic mouse are well separated in their spectral sensitivities (Fig. 2). This allows the possibility that spectral sensitivity may be selectively altered by exposure to lights of different chromatic content. In previous ERG measurements we showed that small shifts ( $\sim 0.10$ log unit) in spectral sensitivity could be produced by intense chromatic adaptation (Shaaban et al., 1998). The effects were of a size consistent with passive pigment bleaching. Here we examined the effects of chromatic adaptation on visual discrimination.

To accomplish this we measured increment thresholds on chromatic backgrounds. Specifically, 500 and $600 \mathrm{~nm}$ lights were used both as test lights and background lights so that a total of four thresholds were measured: two homochromatic and two heterochromatic thresholds. The logic is that if these two lights have no differential influence on spectral sensitivity, then on average the

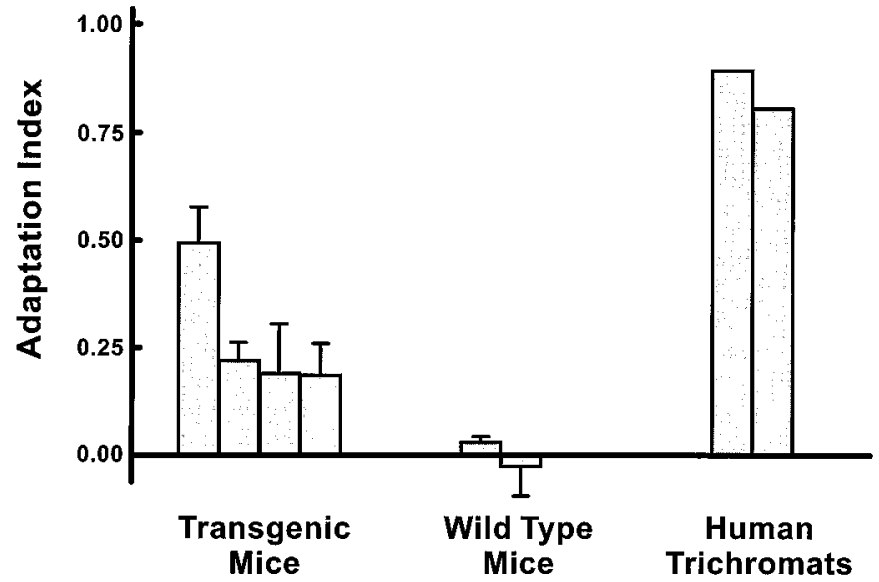

Figure 8. Results from a chromatic adaptation test. The adaptation index is based on a comparison of four thresholds ( 2 heterochromatic, 2 homochromatic) measured in a discrimination task. See Results for details. Values for the mice represent means (error bars represent 1 SD) for five replications of the experiment. Two trichromatic human subjects were tested in a single session.

heterochromatic thresholds will equal the homochromatic thresholds; alternatively, if there is a differential influence, the heterochromatic thresholds will be lower than the homochromatic thresholds (Boynton et al., 1965). The four thresholds were measured using exactly the procedures described for assessing spectral sensitivity (above). The intensity of the $600 \mathrm{~nm}$ background light $\left(109.6 \mathrm{~cd} / \mathrm{m}^{2}\right)$ was set to the brightest value that could be used consistent with our still being able to measure a threshold for a $600 \mathrm{~nm}$ test light. The intensity of $500 \mathrm{~nm}$ background light was then adjusted according to the mouse photopic spectral sensitivity function (Fig. 4) to make it approximately equally effective to the $600 \mathrm{~nm}$ background light. Four transgenic mice were tested. Because there were noticeable variations among animals, a total of five separate threshold measurements were made for each of the four test lights. To provide a comparative baseline, exactly analogous tests were run on two wild-type mice and on two human trichromats.

The results from these tests are summarized in Figure 8. Plotted for each of eight subjects is an index of adaptation, here defined as the difference in average (log) sensitivity between the heterochromatic and homochromatic tests (error bars $=1 \mathrm{SD}$ ). Wild-type mice have only a single cone photopigment active over the 500 to $600 \mathrm{~nm}$ portion of the spectrum. They should thus show no differential chromatic adaptation, and they did not. To the contrary, each of the transgenic mice did show evidence for significant differential adaptation. For three of these animals the magnitude of the effect was roughly similar; for the fourth animal (result plotted leftmost in Fig. 8) the influence of different chromatic backgrounds was consistently larger. Adaptation effects for the transgenic mice were in turn much smaller than that measured for trichromatic humans comparably tested.

\section{Wavelength discrimination}

The presence of two photopigments that can be differentially affected by chromatic adaptation raises the possibility that the visual system of the transgenic mouse might be able to use signals from M- and L-pigments to permit pure-wavelength discrimination, i.e., have color vision. We tested three transgenic mice to determine whether they could make such a wavelength discrimination. For this, the test light was set to $500 \mathrm{~nm}$, and the other two 


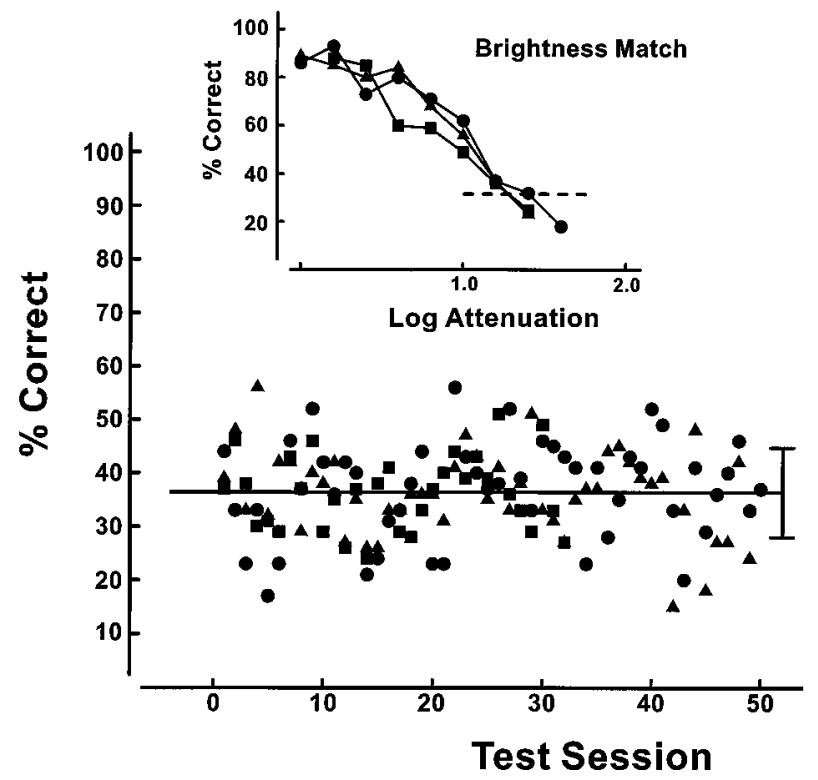

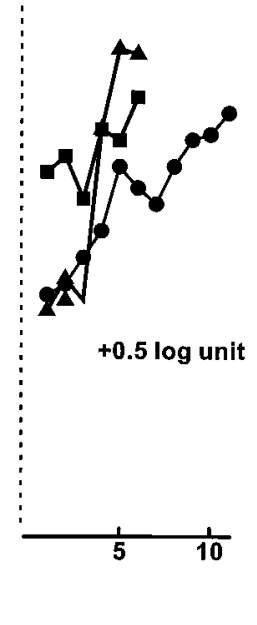

Figure 9. Test for wavelength discrimination in three transgenic mice (separate symbols). The inset at the top shows results from the procedure that was used to establish individual brightness equations for 500 and $600 \mathrm{~nm}$ lights (see Results). Below are the average performance values recorded in daily test sessions in which each mouse was required to discriminate between equally bright 500 and 600 $\mathrm{nm}$ lights. The horizontal line shows the average performance across the test period (vertical bar $=2 \mathrm{SDs}$ ). The performance to the right of the vertical dashed line was recorded subsequent to the addition of a systematic brightness difference ( $0.5 \log$ unit) between the stimulus pair. panels were illuminated with $600 \mathrm{~nm}$ light. Unlike all of the previous discrimination tests, in this experiment the test light was not added to the background light but instead replaced it on each trial. The intensity of the $600 \mathrm{~nm}$ light was the same as in the previous experiment. A first step was to determine the intensity of the $500 \mathrm{~nm}$ test light required to render it equally as bright as the $600 \mathrm{~nm}$ light. The way this was done is summarized in the inset to Figure 9. Each of the three mice had previously participated in several experiments in which they had been reinforced for picking the brighter of three test lights. Consequently, at the point this experiment was initiated they were well trained to select the brighter of two stimuli. To determine a brightness equation, the intensity of a bright $500 \mathrm{~nm}$ light was progressively dimmed in steps of $0.2 \log$ unit. When the $500 \mathrm{~nm}$ light was much brighter than the $600 \mathrm{~nm}$ light, the animal selected it consistently, but as the intensity was decreased the percentage of correct selections declined and eventually reversed, i.e., the animal began to more frequently select the $600 \mathrm{~nm}$ light because it was now, presumably, the brighter of the two. The point at which performance passed through the level of chance (33\%; Fig. 9, dashed line) was taken as the point of equal brightness between the two lights. Note that the equation value was effectively the same for each of the three animals, varying across subjects by $<0.1 \log$ unit.

With the brightness equations established, each animal was tested to determine whether it could learn to successfully discriminate between equally bright 500 and $600 \mathrm{~nm}$ lights. In each daily test session, the two lights were presented at the calculated point of equal brightness and on equal numbers of trials for values that were both $0.2 \log$ unit higher and lower. The average performance for the equal brightness pairings for each test session is shown at the bottom of Figure 9. These tests were continued until it became apparent that the mice were unable to acquire this discrimination. The total number of test trials accumulated for the three subjects varied over the range from 8100 to 13,500 . As can be seen, across all that experience with the pair of test lights there is no deviation from chance performance. To be certain that this failure reflected the specific absence of a capacity for this discrimination, not merely some general decline in performance, a systematic difference ( $0.5 \log$ unit) in the brightness of the two lights was introduced at the end of the test period (shown to the right of the vertical dashed line in Fig. 9). In the presence of this systematic brightness cue, each of the three animals almost immediately began to show successful discrimination. The results of this experiment seem clear-cut: these transgenic mice are incapable of making a pure-wavelength discrimination between 500 and $600 \mathrm{~nm}$ lights.

\section{DISCUSSION}

In recent years transgenic mice have been used to study basic retinal physiology and to provide models for investigating retinal disease. Typically this has been done through expression of mutant genes that influence rods (Lem and Makino, 1996). Our experiments have involved a different approach in that a foreign cone pigment gene was expressed in a transgenic animal for the purpose of examining the extent to which a visual system can incorporate and exploit a new source of environmental information. The results make clear that photons absorbed by a newly acquired photopigment expand significantly the spectral range over which mice can see. An added class of photopigment theoretically could also underlie a new dimension of spectral experience, but that did not occur.

\section{Cone photopigment arrangements and neural organization in transgenic mice}

The retinas of the transgenic mice contain three classes of cone photopigment: the native UV and M-pigments and the transgenic L-pigment (Fig. 2). Previous antibody labeling experiments showed that the L-pigment is coexpressed with the UV and M-pigments in many, although not all, cones of the transgenic mouse (Shaaban et al., 1998). Because of the current ambiguity about the distribution of cone pigments in the wild-type mouse, it is not possible to be more precise about the cones of the transgenic mouse. The history of the issue is as follows. The first opsin-antibody labeling experiments on the mouse retina detected two classes of cone in the mouse retina that had a unique topographic organization (Szel et al., 1992). Cones expressing M-pigment were thought to be restricted to a region of the retina located roughly superior to the horizontal meridian; cones expressing UV pigment were found throughout the retina, but were at highest density in the ventral half of the retina. Later antibody 


$\begin{aligned} & \text { Table 1. Range of cone types in wild-type and transgenic mice as } \\
& \text { defined by photopigment complement }\end{aligned}$
\begin{tabular}{cl} 
Wild type & Transgenic \\
\hline$U V$ & UV \\
& UV + L \\
UV + M & UV + M \\
& UV + M + L \\
& $M+L$ \\
$M$ & $M$ \\
\hline
\end{tabular}

labeling experiments suggested that the mouse retina has a transitional zone between the superior and inferior retinal areas, some 100-500 $\mu \mathrm{m}$ in width, that contains cones coexpressing UV and M-pigments (Rohlich et al., 1994). This implies that there are three populations of cone in the retinas of wild-type mice as defined by their photopigment complements (Table 1), but there is considerable uncertainty about the relative representation of these three cone types. That is because a recent antibody-labeling experiment suggests that cones coexpressing UV and M-pigments are much more widespread than the earlier report indicated (Glosmann and Ahnelt, 1998), perhaps extending over much of the entire retina. That contention receives support in results from an electrophysiological experiment (Lyubarsky et al., 1998). In sum, there are potentially six types of cone in the transgenic mouse that have distinctly differing spectral sensitivities (Table 1 ), but their relative representation and spatial distributions are not known.

Earlier measurements indicated that in transgenic mice of the type used here L-pigment was expressed to a level that accounted for $\sim 77 \%$ of the total of M- and L-pigments (Shaaban et al., 1998). The best summative fit of $M$ and $L$ contributions to the averaged ERG spectral sensitivity curve (Fig. 3) required a similar weighting (L, 86\%), suggesting that the relative representation of the two pigments is straightforwardly reflected in the amplitudes of ERG cone signals. The relationship is thus similar to that seen in the human retina where the $\mathrm{L} / \mathrm{M}$ weighting required to best fit spectral sensitivity curves obtained from the flicker ERG appears to index the relative numbers of $\mathrm{L}$ and $\mathrm{M}$ cones (Jacobs and Neitz, 1993).

The relative influence from L-pigment signals was considerably lessened in the behavioral measurements of spectral sensitivity where the identical fitting procedure yielded a required L-cone contribution of only $36 \%$ of the total of $\mathrm{M}+\mathrm{L}$ (Fig. 4). There are numerous differences inherent in the measurement procedures that could potentially influence the relative contributions of Mand L-pigments to outer retinal signals and behavioral sensitivity: for example, differences in light levels and state of adaptation. One possible key to understanding the relative reweighting of $\mathrm{M}$ and $\mathrm{L}$ influence is that UV cone signals were also represented very differently in ERG and behavioral measurements. Specifically, cones containing UV pigment contribute relatively much less to behavioral spectral sensitivity than they do to the ERG spectral sensitivity functions (Fig. 5, compare top and bottom). Because many cones in the transgenic mouse that contain UV pigment also contain L-pigment, the loss of influence from the two pigment types in the behavioral measures likely may simply reflect a diminution in the influence of the signals from cones containing UV pigment. Whatever the reason for this, and several could be imagined, it is intriguing that apparently robust UV cone signals do not contribute much to mouse vision in this test situation.

Even with modest light levels, significant alterations in behavioral $\mathrm{M} / \mathrm{L}$ spectral sensitivity were produced by chromatic adaptation. These effects were substantially larger than those obtained earlier for analogous ERG measurements made at much higher light levels (Shaaban et al., 1998). A minimal implication of this result is that signals originating from $\mathrm{M}$ - and L-pigments must be represented, at least to some extent, in separable neural pathways at locations beyond the ERG generators. One of the four transgenic mice tested showed significantly larger adaptation effects than did the other three (Fig. 8). This animal was not unusual in any other way; in particular, his spectral sensitivity functions were not extreme in their relative representation of $\mathrm{M}$ - and L-pigment signals.

\section{Absence of $\mathrm{M} / \mathrm{L}$ color vision in transgenic mice}

The minimal requirements for vertebrate color vision include the presence of more than one spectral mechanism and a means for comparing the outputs from these spectral mechanisms somewhere in the nervous system. As far as is now known, the latter is always accomplished through the presence of spectrally opponent cells. From the six presumed receptor types in the transgenic mouse retina (Table 1), there are eight separate pairings that have sufficiently different spectral sensitivity to 500 and $600 \mathrm{~nm}$ lights that they could potentially provide inputs that might allow for color discrimination; in particular, of these, the M versus UV $+\mathrm{L}$ receptors would be expected to provide strikingly different relative sensitivities to the 500 and $600 \mathrm{~nm}$ lights. So a potential receptor basis for $\mathrm{M} / \mathrm{L}$ color vision exists in the transgenic mouse. The details of the neural organization of the mouse retina remain, somewhat surprisingly, relatively unstudied, but there are two facts potentially relevant to the present case. First, many mouse ganglion cells show antagonistic center/surround organizations (Balkema and Pinto, 1982; Stone and Pinto, 1993). If these centers and surrounds segregate different spectral inputs in the transgenic mouse they could provide the information required for the elaboration of $\mathrm{M} / \mathrm{L}$ color vision. Second, a brief report suggests an abundant presence (nearly $20 \%$ of all cells recorded) of spectral opponency among mouse ganglion cells (Yamamoto and Gouras, 1993). The opponency is described as involving short- and middle-wavelength mechanisms. The former are presumably UV cones, and the latter could conceivably be either M cones or rods. Both of these findings allow the possibility that the mouse retina may have a neural organization that could be used to produce novel color vision in the transgenic mouse.

It was against this background of research and our own observation that significant differential chromatic adaptation can be produced in the transgenic mouse that we were encouraged to determine whether these animals had acquired some new color vision. A failure to demonstrate a sensory capacity in discrimination tests requires cautious interpretation. In particular, an alternative test paradigm or training strategy might yield a different outcome. Nevertheless, the present results seem quite convincing: the added L-pigment substantially expands the spectral window of mouse, but it does not allow for any new chromatic discriminations.

The fact that the added cone pigment did not translate into new color vision in the transgenic mouse unexpectedly raises questions about color vision in the wild-type mouse. The reason is as follows. The wild-type mouse might be predicted to have dichromatic color vision, mediated by neural comparison of signals from 
cones containing UV and M-pigments. However, these same receptors and their neural connections should also have provided the substrate for any added $\mathrm{M} / \mathrm{L}$ color vision in the transgenic mouse, e.g., by comparison of $\mathrm{M}$ versus $\mathrm{UV}+\mathrm{L}$ or $\mathrm{M}+\mathrm{UV}$ versus $U V+L$ (Table 1 ). The failure to see $M$ versus $L$ color vision in the transgenic mouse thus raises a doubt about the presence of any significant UV versus $M$ color vision in the wild-type mouse.

\section{Receptor coexpression of photopigments}

It has long been known that numerous species of fish and amphibia can construct photopigments from either of two chromophores, frequently interchanging the two during lifestyle changes such as migration or metamorphosis. These chromophore changes yield shifts in the absorption spectra of photopigments, and a consequence is that individual photoreceptors may contain a pair of pigments having different spectra (Bowmaker, 1991). There are recent indications that multiple pigments can be similarly expressed in a single receptor in some species through the presence of multiple opsins, each of which is complexed to the same chromophore. For instance, microspectrophotometric measurements of cones in the retina of the guppy (Poecilia reticulata) yielded spectra that appear to reflect the combined presence of two photopigments that are peakseparated by nearly $40 \mathrm{~nm}$ (Archer and Lythgoe, 1990), whereas UV-sensitive cones in tiger salamanders (Ambystoma tigrinum) may have as many as three active opsins that allow the photoreceptor to significantly absorb light all the way across the visible spectrum (Makino and Dodd, 1996). Recent antibody-labeling experiments suggest the presence of some cones containing two spectrally discrete photopigments in a number of mammalian species; the mouse (as described here), the guinea pig, and the rabbit retina contain a population of cones that coexpress two different photopigments (Rohlich et al., 1994). All of these examples suggest that the mice that are the focus of this investigation provide a less artificial model than would have been supposed even a few years ago. Our measurements show that coexpression of pigments in single receptors can yield the immediate advantage of an expanded spectral window, so the transgenic mouse may provide a model of a normal step in the evolution of visual systems. Whether that is true or not, the ability to obtain good functional expression of a human photopigment in the mouse opens the door to examination of the consequences of mutated versions of human cone pigment genes in a well defined model system.

\section{REFERENCES}

Archer SN, Lythgoe JN (1990) The visual pigment basis for cone polymorphism in the guppy, Poecilia reticulata. Vision Res 30:225-233.

Asenjo AB, Rim J, Oprian DD (1994) Molecular determinants of human red/green color discrimination. Neuron 12:1131-1138.

Balkema Jr GW, Pinto LH (1982) Electrophysiology of retinal ganglion cells in the mouse: a study of a normally pigmented mouse and a congenic, hypopigmentation mutant, pearl. J Neurophysiol 48:968-980.

Bowmaker JK (1991) Visual pigments, oil droplets and photoreceptors. In: The perception of colour (Gouras P, ed), pp 108-127. Boca Raton, FL: CRC.

Boynton RM, Scheibner H, Yates T, Rinalducci E (1965) Theory and experiments concerning the heterochromatic threshold-reduction factor (HTRF). J Opt Soc Am A 55:1672-1685.

Dawis SM (1981) Polynomial expressions of pigment nomograms. Vision Res 21:1427-1430.
Deeb SS, Motulsky AG (1996) Molecular genetics of human color vision. Behav Genet 26:195-207.

Douglas RH, Partridge JC, Marshall NJ (1998) The eyes of deep-sea fish. I: Lens pigmentation, tapeta and visual pigments. Prog Retinal Eye Res 17:597-636.

Glosmann M, Ahnelt PK (1998) Coexpression of M- and S-opsin extends over the entire inferior mouse retina. Invest Ophthalmol Vis Sci 39:S1059.

Jacobs GH (1983) Within-species variations in visual capacity among squirrel monkeys (Saimiri sciureus): sensitivity differences. Vision Res 23:239-248

Jacobs GH (1984) Within-species variations in visual capacity among squirrel monkeys (Saimiri sciureus): color vision. Vision Res 24:1267-1277.

Jacobs GH (1993) The distribution and nature of colour vision among the mammals. Biol Rev 68:413-471.

Jacobs GH (1998) A perspective on color vision in platyrrhine monkeys. Vision Res 38:3307-3313.

Jacobs GH, Neitz J (1993) Electrophysiological estimates of individual variation in the $\mathrm{L} / \mathrm{M}$ cone ratio. In: Colour vision deficiencies $\mathrm{XI}$ (Drum B, ed), pp 107-112. Dordrecht: Kluwer.

Jacobs GH, Neitz J, Deegan II JF (1991) Retinal receptors in rodents maximally sensitive to ultraviolet light. Nature 353:655-656.

Jacobs GH, Neitz J, Krogh K (1996) Electroretinogram flicker photometry and its applications. J Opt Soc Am A 13:641-648.

Lem J, Makino CL (1996) Phototransduction in transgenic mice. Curr Opin Neurobiol 6:453-458.

Lyubarsky AL, Falsini B, Pennesi ME, Valentini P, Pugh Jr EN (1998) UV- and midwave-sensitive cone-driven retinal responses of the mouse: a possible phenotype for coexpression of cone photopigments. J Neurosci 19:442-455.

Makino CL, Dodd RL (1996) Multiple visual pigments in a photoreceptor of the salamander retina. J Gen Physiol 108:27-34.

Merbs SL, Nathans J (1993) Role of hydroxyl-bearing amino acids in differentially tuning the absorption spectra of the human red and green cone pigments. Photochem Photobiol 58:706-710.

Nathans J (1987) Molecular biology of visual pigments. Annu Rev Neurosci 10:163-194.

Neitz M, Neitz J (1998) Molecular genetics and the biological basis of color vision. In: Color vision-perspectives from different disciplines (Backhaus WGK, Kliegl R, Werner JS, eds), pp 101-119. Berlin: Walter de Gruyter.

Neitz J, Neitz M, Jacobs GH (1993) More than three different cone pigments among people with normal color vision. Vision Res 33:117-122.

Olsson JE, Gordon JW, Pawlyk BS, Roof D, Hayes A, Molday RS, Mukai S, Cowley GS, Berson EL, Dryja TP (1992) Transgenic mice with a rhodopsin mutation (Pro23His): a mouse model of autosomal dominant retinitis pigmentosa. Neuron 9:815-830.

Palacios AG, Varela FJ, Srivastava R, Goldsmith TH (1998) Spectral sensitivity of cones in the goldfish, Carassius auratus. Vision Res 38:2135-2146.

Remtulla S, Hallett PE (1985) A schematic eye for the mouse, and comparisons with the rat. Vision Res 25:21-31.

Rohlich P, van Veen T, Szel A (1994) Two different visual pigments in one retinal cone cell. Neuron 13:1159-1166.

Shaaban SA, Crognale MA, Calderone JB, Huang J, Jacobs GH, Deeb SS (1998) Transgenic mice expressing a functional human photopigment. Invest Ophthalmol Vis Sci 39:1036-1043.

Stone C, Pinto LH (1993) Response properties of ganglion cells in the isolated mouse retina. Vis Neurosci 10:31-39.

Szel A, Rohlich P, Caffe AR, Juliusson B, Aguire G, Van Veen T (1992) Unique separation of two spectral classes of cones in the mouse retina. J Comp Neurol 325:327-342.

Winderickx J, Lindsey DT, Sanocki E, Teller DY, Motulsky AG, Deeb SS (1992) Polymorphism in red photopigment underlies variation in colour matching. Nature 356:431-433.

Yamamoto S, Gouras P (1993) Color opponent neurons in mouse retina. Soc Neurosci Abstr 19:1257. 